Pensamiento Crítico Vol. 20 No 2, pp. 53-68

\title{
Determinantes de las exportaciones no tradicionales en el Perú 2002 - 2015
}

\author{
Determining the non-traditional exports in Perú 2002 - 2015
}

[Recepción: Setiembre de 2015 / Conformidad: Octubre de 2015]

Rafael Bustamante Romani ${ }^{1}$

\section{RESUMEN}

En el presente estudio se estima una función de demanda de exportaciones no tradicionales para el caso peruano utilizando análisis multivariado de cointegración multivariado, el cual examina la existencia de una relación de largo plazo entre las exportaciones no tradicionales, la demanda externa, la demanda interna, el nivel de empleo y el índice de tipo de cambio real bilateral y multilateral. Los resultados muestran que la demanda del exterior juega un papel considerable en la determinación de las exportaciones no tradicionales en Perú. Igualmente, el tipo de cambio real bilateral y multilateral también afecta de forma significativa a la demanda de exportaciones no tradicionales. La interpretación de las respectivas elasticidades comprende la obtención de ellas a partir del vector de cointegración.

Palabras clave: Cointegración, exportaciones, exportaciones no tradicionales.

1 Docente investigador de la UNMSM. Cursos de enseñanza: Econometría Financiera, Microeconometría, Macroeconometría, Valorización de empresas, estadística para la toma de Decisiones Empresariales. MBA CENTRUM, PUCP. Con estudios de Doctorado en Economía UNAM, México.

Este documento está basado en: "Exportaciones no Tradicionales en Colombia y sus Determinantes". Martha Misas A, María Teresa Ramirez G, Luisa Fernanda Silva. 


\title{
Pensamiento Crítico Vol. 20. No 2
}

\begin{abstract}
In the present study is estimated a function of demand for non- traditional exports for Peru multivariate analysis using multivariate cointegration, which examines the existence of a long-term relationship between non-traditional exports, external demand, domestic demand is estimated, the level of employment and the rate of the bilateral and multilateral real change. The results show that foreign demand plays a significant role in the determination of non-traditional exports in Peru. Similarly, the bilateral and multilateral real exchange rate also significantly affect the demand for nontraditional exports. The interpretation of the respective elasticities comprises obtaining the same from cointegration.
\end{abstract}

Keywords: Cointegration, exports, non-traditional exports.

\section{Introducción}

La relación entre las exportaciones no tradicionales en Perú y sus determinantes sigue siendo un tema importante para la investigación. En el contexto internacional, la mayoría de estudios a nivel mundial encuentra una relación significativa entre las exportaciones no tradicionales y el tipo de cambio real. Sin embargo, el rango de la elasticidad de dicha relación varía considerablemente entre los diferentes estudios. Estudios recientes que han incorporado la demanda externa como determinante de las exportaciones no han encontrado una relación significativa entre ellas (Mesa, Cock, \& Jiménez).

Con el objeto de estudiar este sector, analizamos los determinantes de las exportaciones no tradicionales. Para ello, se estima una función de demanda utilizando análisis multivariado de cointegración, donde se busca examinar la existencia de una relación de largo plazo entre las exportaciones no tradicionales, la demanda externa del resto del mundo ${ }^{2}$ y los precios relativos internacionales y estimar el mecanismo de corrección de error correspondiente que permita analizar, al mismo tiempo, el impacto del corto plazo ante los shocks que afecten nuestras exportaciones (Reinhart, 1995).

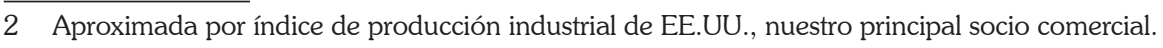




\section{Rafael Bustamante Romaní}

El propósito de este trabajo es estudiar y ponderar las posibles relaciones existentes entre el nivel de las exportaciones no tradicionales peruanas totales, con la demanda externa del resto del mundo, el nivel de empleo y el índice de tasa de cambio real bilateral o multilateral, según corresponda.

\section{Planteamiento del problema}

La mayoría de estos estudios a nivel mundial encuentra una relación significativa entre las exportaciones no tradicionales y el tipo de cambio real. Sin embargo, el rango de la elasticidad de dicha relación varía considerablemente entre los diferentes estudios. De otra parte, el papel de la demanda externa como determinante de las exportaciones no tradicionales en Perú es un tema que debe ser tocado con profundidad. Un grupo de trabajos referentes supone una demanda mundial completamente elástica y, por lo tanto, proceden a estimar funciones de oferta (Villar, L, 1984), (Botero, C.H; Meisel, A.; 1988) y (Alonso, 1993). Por otro lado, estudios recientes que han incorporado la demanda externa como determinante de las exportaciones no han encontrado una relación significativa entre ellas (Mesa, F; Cock , M.; Jiménez , A., 1997).

\section{Hechos estilizados}

Con el propósito de entender los determinantes de las exportaciones no tradicionales en Perú, se considera importante presentar la evolución de estas en el período bajo análisis. En los últimos 10 años (1996-2014), las exportaciones no tradicionales crecieron 231 por ciento en términos nominales y 188 por ciento en términos reales. Asimismo el 52 por ciento de este crecimiento se explica por el aumento de las exportaciones textiles y agroindustriales. Cabe resaltar, sin embargo, que se han registrado crecimientos cercanos o mayores del 100 por ciento en la mayoría de sectores. A continuación, en la figura 1, se presenta la evolución de las exportaciones a partir del año 1990. 


\section{Pensamiento Crítico Vol. 20. № 2}

FIGURA 1

Exportaciones de productos no tradicionales

(Millones de US\$)

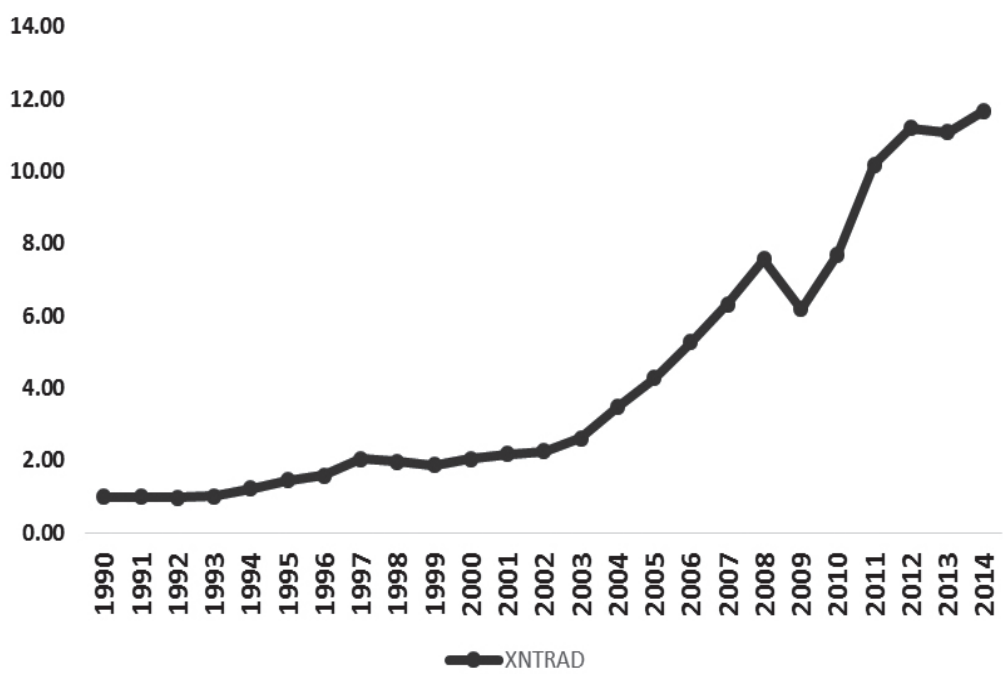

Fuente: BCRP. Elaboración del autor.

Asimismo el número de empresas exportadoras ha aumentado 51 por ciento entre 2001 y 2006, mientras que aquellas con exportaciones superiores a US\$ 30 millones aumentaron en 343 por ciento en el mismo período. La diversificación ha explicado un alto porcentaje del crecimiento de las exportaciones en los últimos diez años: el 55,5 por ciento de las exportaciones no tradicionales del 2006 se explicó por la exportación de productos no exportados en 1996 o hacia países a los que no se exportó en dicho año. Al respecto, el crecimiento de las exportaciones, explicado por la aparición de nuevos productos, es particularmente importante en la segunda mitad de los noventa. Durante la presente década, el aumento de las exportaciones se explica básicamente por el crecimiento de las ventas de los productos ya exportados.

Los principales países de destino de nuestras exportaciones fueron Estados Unidos de América con 19,9\%, China 12,6\%, Chile 4,7\%, Japón 4,6\% y Venezuela con $4,6 \%$ del valor total de las exportaciones. 


\section{Rafael Bustamante Romaní}

\section{FIGURA 2}

Composición de las exportaciones totales

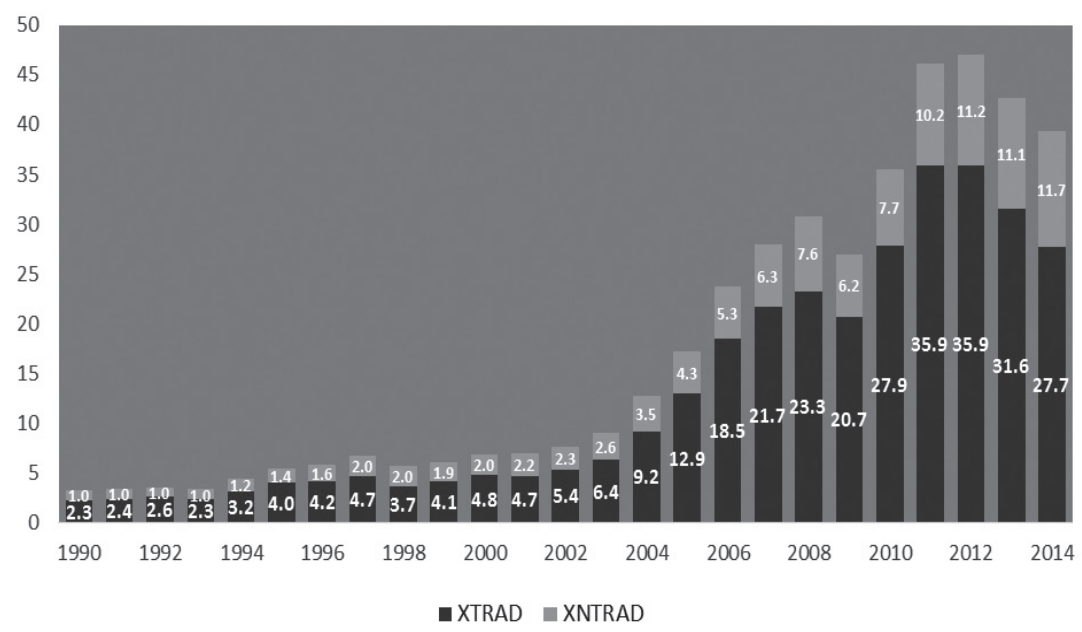

Fuente: BCRP. Elaboración del autor.

En la figura 2 se presenta la evolución de las exportaciones peruanas totales en términos de su composición: exportaciones tradicionales vs. exportaciones no tradicionales.

\section{Marco teórico}

A partir de los estudios de Reinhart $(1995)^{3}$ se plantea la demanda de exportaciones no tradicionales del país doméstico, en este caso Perú; se puede derivar desde la perspectiva del país extranjero, en este caso Estados Unidos, a través de la maximización de una función de utilidad intertemporal de un agente representativo en el país extranjero. Este agente representativo consume bienes no transables producidos en el país extranjero $(\mathrm{N})$ y bienes importados, los cuales corresponden a las exportaciones del país doméstico, es decir, Perú (XNT). Así, la función de utilidad intertemporal puede ser expresada como:

3 La presentación y desarrollo del modelo en este artículo sigue de cerca a C. Reinhart (1995). 


\section{Pensamiento Crítico Vol. 20. No 2}

$$
U=\int_{0}^{\infty} e^{\beta t} u\left(N_{t}, X N T_{t}\right) d t
$$

Donde $0<\beta<1$ representa una tasa constante de descuento, dado que todos los argumentos de la función de utilidad están medidos en términos reales.

La ecuación a ser estimada se plantea a través de la siguiente relación:

$$
X N T=\beta_{0}+\beta_{1} r_{t}+\beta_{2} R_{t}+\varepsilon_{t}
$$

La ecuación (12) presenta una especificación tradicional del equilibrio de largo plazo de la demanda de exportaciones ${ }^{4}$. De tal forma que, a largo plazo, cualquier desviación entre los valores observados y esperados de las exportaciones reales tiende a desaparecer, es decir $X N T=X N T^{\text {esperadas }}$.

En particular, los determinantes básicos del logaritmo de las exportaciones reales deseadas, $X N T^{\text {esperadas }}$, son los logaritmos de los precios relativos, cuya proxy es el índice del tipo de cambio real, $r_{t}$, y una variable de escala que captura las condiciones del ingreso mundial o demanda mundial ${ }^{5} R_{t}$. Donde un aumento en los precios relativos como un incremento en la actividad económica mundial se reflejarán en un aumento en la demanda de exportaciones reales. Así, se espera que $\beta_{1}>0$ y $\beta_{2}>0$.

Finalmente incluiremos la tasa de empleo como un factor determinante de las exportaciones no tradicionales, es decir, dado que para un mayor nivel de empleo (l) está asociado con la demanda laboral de parte de las empresas exportadoras de productos tradicionales y no tradicionales. Asimismo, como indicadores de competitividad incluiremos el índice del tipo de cambio real bilateral ( $\mathrm{rb}$ ) y el multilateral ( $\mathrm{rm}$ ), con el objeto de tener una visión más amplia del efecto del tipo de cambio real y el producto bruto interno (PBI) como indicador de la demanda interna. Finalmente, nuestra ecuación a estudiar y estimar será:

4 Una especificación similar se tiene al considerar el logaritmo del volumen deseado de exportaciones: $Q^{d}$ Así, la ecuación (11) puede formularse como: $Q^{d}=\mu+\beta_{1} P_{1}+\beta_{2} P+\beta_{3} W_{1}+\varepsilon_{t}$.

5 La demanda de un país específico. 


$$
X N T=\beta_{0}+\beta_{1} r b_{t}+\beta_{2} r m_{t}+\beta_{3} R_{t}+\beta_{4} p b i_{t}+\beta_{5} l_{t}+\varepsilon_{t}
$$

\section{Modelo econométrico y datos}

En esta parte del documento se describe y desarrolla la parte de test de cointegración de Johansen para sistemas de vectores autorregresivos (VAR), con la finalidad de conocer si existe alguna relación de largo plazo entre las series en estudio. La muestra serán todas las variables especificadas en el presente estudio con periodicidad mensual para los años 2002- 2014.

La información considerada en el primer grupo está conformada por las exportaciones reales no tradicionales totales hacia el resto del mundo, expresada en logaritmos (LNXNT).

Dada la importancia de los Estados Unidos en el comercio exterior de Perú, consideramos a este conjunto de exportaciones reales no tradicionales como las dirigidas al resto del mundo. Este, a su vez, es aproximado por el índice de producción industrial de ese país expresado en logaritmos (LNIPRODIND). En el caso de los precios relativos, se utiliza como proxy el índice de tipo de cambio real bilateral con Estados Unidos (LNITCRB) y el índice del tipo de cambio real multilateral (LNITCRM), ambos expresados en logaritmos.

\subsection{Análisis de los datos}

Realizamos la prueba de raíces unitarias. Para ello utilizamos la prueba de DFGLS, que usa la prueba t de Dickey-Fuller modificada, propuesta por Elliott, Rothenberg y Stock (1996). La esencia de la prueba es hacer una secuencia similar a la prueba de Dickey-Fuller, excepto que la serie de tiempo es transformada a través de una regresión por mínimos cuadrados generalizados (GLS). Elliott, Rothenberg y Stock han demostrado que esta prueba tiene mayor poder que las versiones anteriores de la prueba Dickey-Fuller aumentada ${ }^{6}$. Según este test, vemos que para todas las series en estudio se rechaza la hipótesis nula de existencia de raíz unitaria, dado que el estadístico DFGLS es mayor que el P-estadístico al 5\% de nivel de significancia (Bustamante, 2014).

6 Ver http://www.learneconometrics.com/class/5263/notes/DF.pdf 
Pensamiento Crítico Vol. 20. No 2

\section{CUADRO 1}

\begin{tabular}{lcc}
\hline HO: La serie tiene raiz unitaria & Elliott-Rothenberg-Stock test statistic & P-Statistic \\
\hline \hline LNITCRB & 21.1 & 5.7 \\
LNITCRBM & 13.4 & 5.7 \\
LNPBI & 13.5 & 5.7 \\
LNEMPLEO & 19.3 & 5.7 \\
LNXNT & 23.9 & 5.7 \\
LNIPROD & 4.1 & 5.7 \\
\hline
\end{tabular}

Estimaciones econométricas del autor.

En el cuadro 1 claramente se observa que las series son integradas de orden uno, dado que el estadístico Elliot-Rothenberg-Stock es mayor que el P - Statistic al 5\% de nivel de significancia.

\subsection{Causalidad de Granger}

El test de causalidad de Granger permite comprobar si las series tienen relación unidireccional o bidireccional. Del contraste de causalidad Engle Granger por bloques, a partir de un sistema VAR con cuatro rezagos, se obtiene que todas las variables expuestas como determinantes causan a las exportaciones no tradicionales totales en forma conjunta, a pesar de que algunas a nivel de $5 \%$ de significancia (Bustamante Romaní, 2014).

\section{CUADRO 2}

\begin{tabular}{lccc}
\hline \multicolumn{4}{l}{$\begin{array}{l}\text { VEC Granger Causality/Block Exogeneity Wald Tests } \\
\text { Dependent variable: D(LNXNT) }\end{array}$} \\
\hline \multicolumn{1}{c}{ Excluded } & Chi-sq & df & Prob. \\
D(LNITCRM) & 9.14 & 10 & 0.52 \\
D(LNITCRB) & 9.60 & 10 & 0.48 \\
D(LNIPRODIND) & 8.31 & 10 & 0.60 \\
D(LNPBI) & 11.75 & 10 & 0.30 \\
D(LNEMPLEO) & 9.55 & 10 & 0.48 \\
All & $\mathbf{7 8 . 5 4}$ & $\mathbf{5 0}$ & $\mathbf{0 . 0 1}$ \\
\hline
\end{tabular}

Estimaciones econométricas del autor. 


\section{Rafael Bustamante Romaní}

En el cuadro 2 se observa que la hipótesis nula de no causalidad es rechazada al $5 \%$ de nivel de significancia, dado que el nivel de probabilidad es menor (1\%).

\subsection{Análisis de cointegración}

El equilibrio que nos interesa es aquel generado por series no estacionarias. Se busca el desarrollo de una técnica de estimación que recoja este tipo de relaciones, con el fin de evitar la pérdida de información que se produce de diferenciar las series:

$$
\Delta L N X N T_{t}=\rho_{Y}\left(L N X N T_{t-1}-\beta_{X} X_{t-1}-\beta_{w} w_{t-1}\right)+\sum_{i=1}^{p} a_{Y 1}(i) \Delta Y_{t-i}+\sum_{i=1}^{p} a_{X 1}(i) \Delta X_{t-i}+\sum_{i=1}^{p} a_{W 1}(i) \Delta W_{t-i}+\varepsilon_{y t}
$$

El procedimiento propuesto por Johansen realiza la estimación de manera conjunta de todo el sistema, logrando calcular los coeficientes de toda la relación de cointegración. Para ello se estima un VAR reducido con las variables en niveles para tener el retardo óptimo, esencial por cuanto es la base para calcular el número de vectores de cointegración. Este no debe ser ni muy corto ni muy largo, pues si el retardo es muy corto probablemente no se capture la dinámica del sistema, mientras que si es muy grande se corre el riesgo de perder grados de libertad ${ }^{7}$.

De acuerdo al test elegimos el criterio más robusto, el Test LR, el cual nos indica que el número de rezagos óptimo es 4 , con el cual vamos a estimar la ecuación de cointegración.

Luego de encontrar la mejor especificación posible, se realizan las pruebas de cointegración. Estas se basarán en la prueba de máximo valor propio a un nivel de significancia del $5 \%$.

La selección del modelo para el análisis de cointegración se basó en el hecho de que algunas de las variables consideradas en el vector de cointegración presentan tendencia en sus niveles, y por esta razón es necesario considerar una relación

7 Las herramientas para seleccionar el retardo óptimo es mediante los criterios AIC (criterio de información de Akaike), SC (criterio de información de Schwarz), HQ (criterio de información de Hannan Quinn), FPE (predicción final del error). 


\section{Pensamiento Crítico Vol. 20. No 2}

cointegrante que permita captar dicho fenómeno. El modelo seleccionado bajo las anteriores premisas es el que presenta: intercepto y tendencia lineal en el vector cointegrante, pero no en la parte de corrección de errores.

CUADRO 3

\begin{tabular}{|c|c|c|c|c|}
\hline \multicolumn{5}{|c|}{$\begin{array}{l}\text { Series: LNXNT LNITCRM LNITCRB LNIPRODIND LNPBI LNEMPLEO } \\
\text { Lags interval (in first differences): } 1 \text { to } 4\end{array}$} \\
\hline Hypothesized & & Max-Eigen & 0.05 & \\
\hline No. of CE(s) & Eigenvalue & Statistic & Critical Value & Prob.** \\
\hline None * & 0.32 & 50.17 & 40.96 & 0.00 \\
\hline At most 1 & 0.21 & 30.83 & 34.81 & 0.14 \\
\hline At most 2 & 0.15 & 22.00 & 28.59 & 0.27 \\
\hline At most 3 & 0.09 & 12.45 & 22.30 & 0.61 \\
\hline At most 4 & 0.08 & 10.57 & 15.89 & 0.29 \\
\hline At most 5 & 0.05 & 6.49 & 9.16 & 0.16 \\
\hline
\end{tabular}

Max-eigenvalue test indicates 1 cointegrating eqn(s) at the 0.05 level

* denotes rejection of the hypothesis at the 0.05 level

**MacKinnon-Haug-Michelis (1999) p-values

Estimaciones econométricas del autor

Podemos observar que, de acuerdo a la prueba maximum eigenvalue, existe solo un vector de cointegración al rechazar la hipótesis nula de la no existencia de vectores de cointegración con una probabilidad de 0.00 y no poder rechazar la hipótesis nula de la existencia de un solo vector de cointegración con una probabilidad de $14 \%$ mayor del $5 \%$ de nivel de significancia.

A continuación se presenta el vector de cointegración normalizado y se observa como no todos los coeficientes presentan los signos esperados según el enfoque de elasticidades, es decir, una depreciación del tipo de cambio real bilateral ocasiona una caída en las exportaciones no tradicionales, en -14.6 por ciento. Esto se debería a un efecto hoja de balance dado que los exportadores importan bienes de capital e insumos en dólares y el incremento del tipo de cambio les genera más costos adicionales que los ingresos adicionales que tienen por exportar. Asimismo un aumento del ingreso foráneo del $1 \%$ tiene un impacto positivo en las exportaciones no tradicionales de un $6.1 \%$, pero 


\section{Rafael Bustamante Romaní}

un aumento del ingreso doméstico en un 1\% ocasiona una reducción de esta en -27 \%. De otro lado, se ve que el empleo tiene una fuerte influencia en el nivel de exportaciones no tradicionales. Este hallazgo nos muestra la importancia de fomentar este sector que viene siendo generador de más empleo.

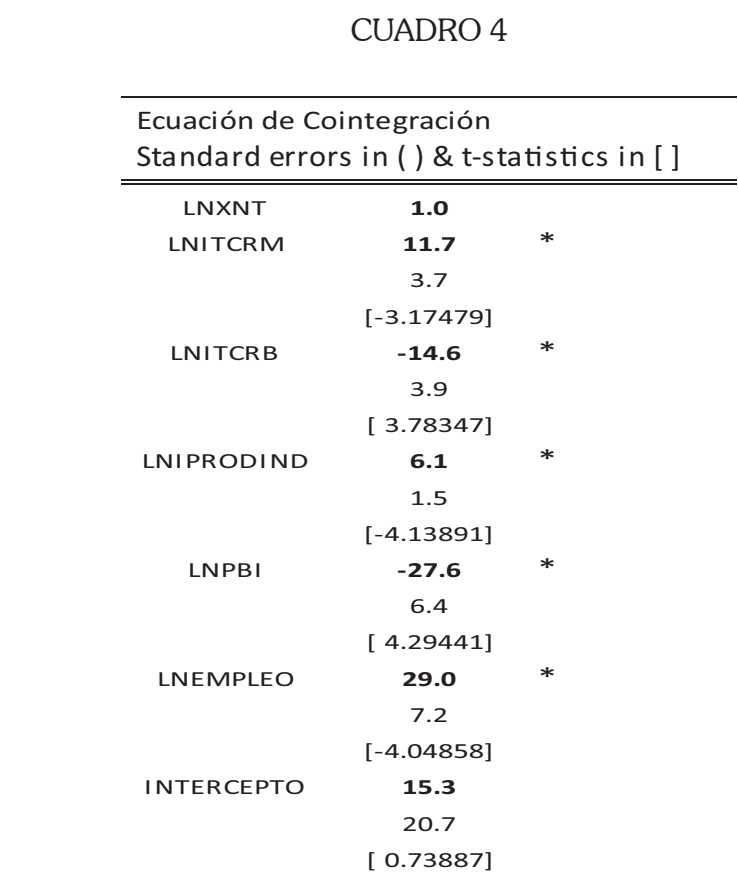

* Indica que el coeficiente es significativo al 5\% de nivel de significancia

Se puede observar que todos los coeficientes de la ecuación de cointegración son significativos a excepción del intercepto.

\subsection{Análisis de la dinámica de corto plazo}

La dinámica de corto plazo se estudia a través de las funciones impulso respuesta generada a partir del modelo de corrección de errores (VEC), estimada en la etapa previa a partir del vector de cointegración. 


\section{Pensamiento Crítico Vol. 20. № 2}

FIGURA 3

Gráfica de la función impulso respuesta

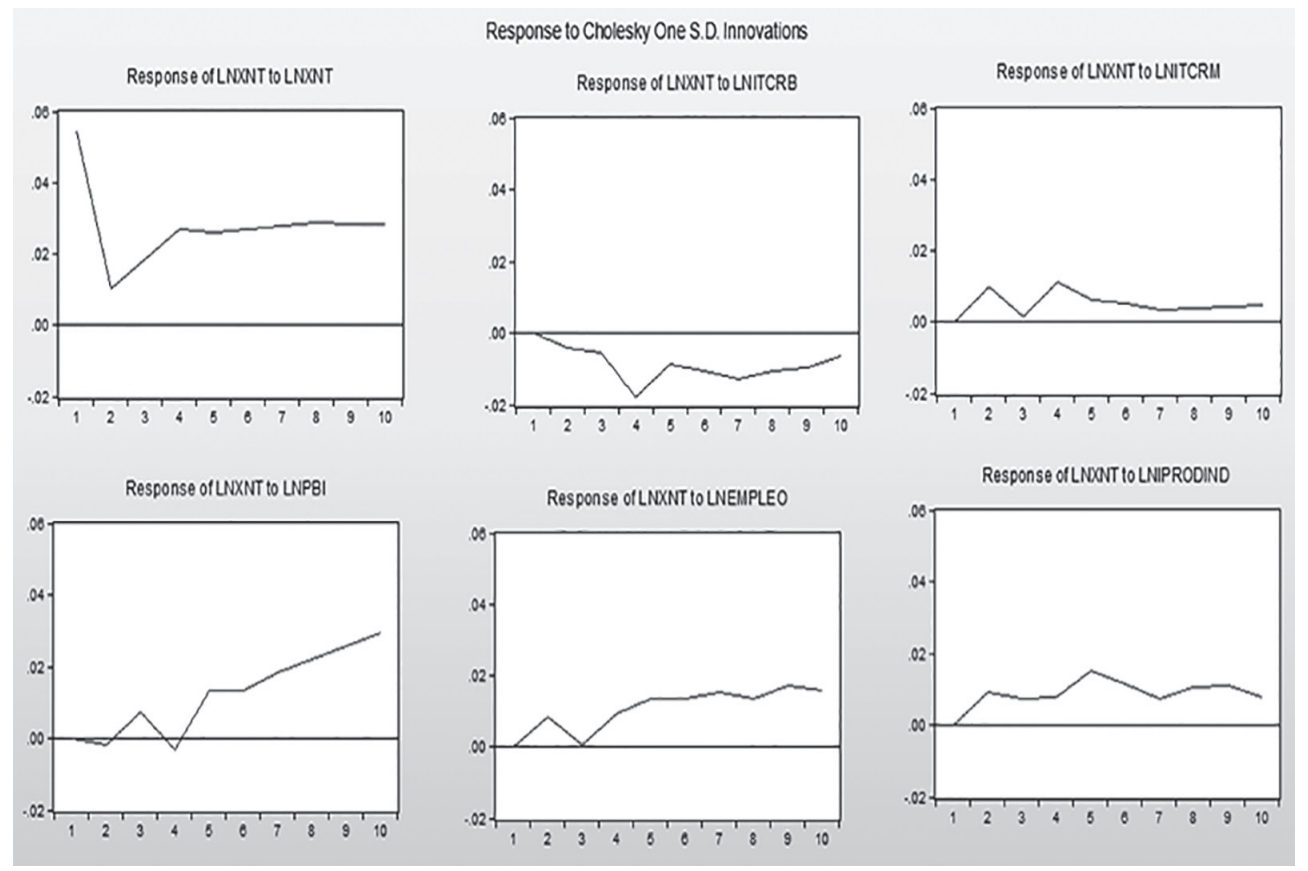

La figura 3 nos muestra que las exportaciones no tradicionales responden de forma negativa ante una depreciación del tipo de cambio real bilateral, pero lo hacen en forma positiva si la depreciación viene por el lado del tipo de cambio real multilateral, efecto que se disipa en aproximadamente un año.

Con respecto al nivel de empleo y el índice de producción industrial de EE.UU., se puede observar que la relación es directa y tiene un efecto permanente en las exportaciones no tradicionales, lo cual puede llevar a conclusiones importantes en el campo de políticas fiscales para promover el crecimiento económico vía exportaciones no tradicionales. 


\section{Rafael Bustamante Romaní}

\subsection{Análisis de la descomposición de varianza}

La descomposición de la varianza es un estudio complementario al análisis impulso-respuesta que informa en distintos horizontes del tiempo el porcentaje de volatilidad que registra una variable por los choques de las demás. Es decir, indica la proporción del efecto que, en forma dinámica, tienen todas las perturbaciones de las variables sobre las demás. Separa la varianza del error de pronóstico para cada una en componentes que pueden atribuirse a cada una de las variables endógenas. De esta manera, es posible medir la volatilidad que le genera la variable endógena a la exógena en un momento específico (Bustamante Romaní, 2014).

\section{CUADRO 5}

Análisis de descomposición de varianza para el PBI

\begin{tabular}{cccccccc}
\hline Period & S.E. & LNXNT & LNITCRM & LNITCRB & LNIPRODIND & LNPBI & LNEMPLEO \\
\hline \hline 1 & 0.05 & 100.00 & 0.00 & 0.00 & 0.00 & 0.00 & 0.00 \\
2 & 0.06 & 91.79 & 2.82 & 0.52 & 2.63 & 0.08 & 2.16 \\
3 & 0.06 & 89.00 & 2.53 & 1.31 & 3.79 & 1.49 & 1.89 \\
4 & 0.07 & 79.99 & 4.38 & 7.21 & 4.01 & 1.29 & 3.13 \\
5 & 0.08 & 73.63 & 4.03 & 6.81 & 6.63 & 3.71 & 5.19 \\
6 & 0.09 & 70.17 & 3.65 & 7.10 & 7.10 & 5.36 & 6.62 \\
7 & 0.10 & 66.69 & 3.18 & 7.63 & 6.46 & 8.01 & 8.02 \\
8 & 0.11 & 63.70 & 2.80 & 7.43 & 6.46 & 11.20 & 8.41 \\
9 & 0.11 & 60.21 & 2.51 & 7.00 & 6.40 & 14.51 & 9.37 \\
10 & 0.12 & 57.42 & 2.32 & 6.33 & 5.96 & 18.19 & 9.78 \\
& Cholesky Ordering: LNXNT LNITCRM LNITCRB LNIPRODIND LNPBI LNEMPLEO \\
\hline
\end{tabular}

En el cuadro 5 se resumen los resultados para el primer y décimo periodo. Se observa que todas las exportaciones no tradicionales (LNXNT) tienen un fuerte comportamiento autorregresivo, en virtud de que, después de 10 meses, más del $50 \%$ de la varianza se sigue explicando por ella misma. 


\section{Pensamiento Crítico Vol. 20. N²}

\section{Conclusiones y recomendaciones}

Los resultados empíricos confirman la existencia de una relación de largo plazo entre las exportaciones no tradicionales, el índice del tipo de cambio bilateral y multilateral, la demanda interna y la demanda externa.

Una depreciación del tipo de cambio real bilateral ocasiona una caída en las exportaciones no tradicionales, en -14.6 por ciento. Esto se debería a un efecto hoja de balance, dado que los exportadores importan bienes de capital $e$ insumos en dólares y el incremento del tipo de cambio les genera más costos adicionales que los ingresos adicionales que tienen por exportar productos con poco valor agregado. Asimismo, un aumento del ingreso foráneo del $1 \%$ tiene un impacto positivo en las exportaciones no tradicionales de un $6.1 \%$, pero un aumento del ingreso doméstico (LNPBI) en un $1 \%$ ocasiona una reducción de esta en $-27 \%$. De otro lado se ve que el empleo (LNEMPLEO) tiene una fuerte influencia en el nivel de exportaciones no tradicionales, este hallazgo nos muestra la importancia de fomentar este sector generador de empleo.

Asimismo, podemos señalar que un buen indicador de competitividad en términos de precios relativos de este sector es el tipo de cambio real multilateral, el cual tiene un efecto bastante considerable sobre las exportaciones no tradicionales, esto debido a un incremento del mismo en $1 \%$, genera un incremento de las exportaciones no tradicionales de $11.7 \%$. Esta evidencia nos muestra la importancia que tiene fomentar el sector para reducir la tasa de desempleo de nuestra economía.

A pesar del crecimiento de nuestras exportaciones, el Perú sigue cosechando sus mayores frutos en productos de exportación tradicionales como la minería. Situación que es problemática en tanto nos hace dependientes de unos pocos productos (cobre, estaño, hierro, oro, etc.), cuyos precios no son estables en el mercado internacional.

Además de los problemas ambientales causados por la minería, está también el hecho de que el sector minero no suele generar encadenamiento. Esto quiere decir que el sector minero opera de forma relativamente aislada. Por ello, no genera mayores beneficios para otros sectores de la economía. 


\section{Rafael Bustamante Romaní}

Para generar encadenamiento es necesario pasar de la exportación de materias primas a productos elaborados, es decir, con valor agregado. Países como Japón o Alemania son ricos a pesar de no tener materias primas en abundancia. Allí, la materia prima es el conocimiento que se traduce en empresas que desarrollan tecnología de punta.

Asimismo, la importancia de la educación de calidad para el futuro, reflejado en la educación básica de las escuelas, institutos y en las universidades, puede llevarnos de una economía dependiente de materias primas a una exportadora de ideas y productos innovadores con alto valor agregado.

\section{Referencias bibliográficas}

Alonso, G. (1993). La oferta de exportaciones menores en Colombia,1970-1992. Coyuntura Económica, XXIII(2).

Botero, C.H; Meisel, A. (1988). Funciones de oferta de las exportaciones menores colombianas. Ensayos sobre Política Económica.

Bustamante Romaní, R. (2014). Vectores autorregresivos. UNMSM, Economía. Obtenido de http://economia.unmsm.edu.pe/data/apu_cla/Apuntes_de_Clase_Nro2_Bustamante.pdf

Bustamante, R. R. (2014). Análisis de Series de Tiempo. UNMSM, Economía. Obtenido de http://economia.unmsm.edu.pe/data/apu_cla/Apuntes_de_Clase_Nro4_Bustamante.pdf

Mesa, F., Cock, A., \& Jiménez, A. (s.f.).

Mesa, F; Cock , M.; Jiménez , A. (1997). Evaluación teoríca y empírica de la exportaciones no tradicionales en Colombia. Revista de Economía del Rosario.

Reinhart, C. (1995). "Devaluation, Relative Prices, and International Trade Evidence from Developing Countries. (I. S. Papers, Ed.) 42(2). 


\section{Pensamiento Crítico Vol. 20. No 2}

Villar, L. (1984). Determinantes de la evolución de las exportaciones menores en Colombia 1960-1981. Coyuntura Económica, 15(3).

Villar, L. (1984). Determinantes de la evolución de las exportaciones, 1960-1981. Coyuntura Económica, XIV(3). 